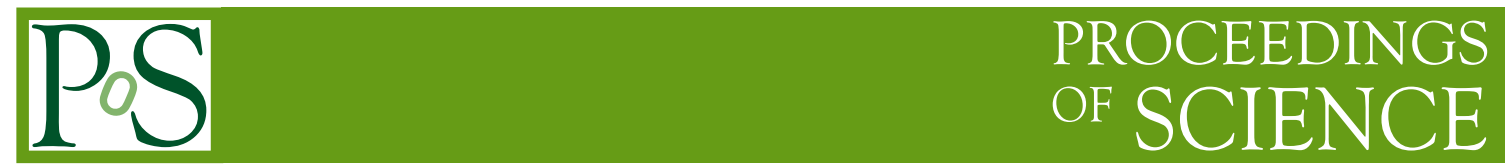

\title{
Search for Pair Production of Leptoquarks in the CMS Experiment
}

\author{
Paolo Rumerio on Behalf of the CMS Collaboration \\ University of Maryland \\ E-mail: rumerio@cern.ch
}

Leptoquarks are hypothetical particles carrying both lepton and quark flavor. They are predicted by Grand Unified Theories, technicolor and composite models, among others. First results from the searches for pair production of first- and second-generation scalar leptoquarks at the CMS experiment using the entire 2010 proton-proton collision data at $\sqrt{s}=7 \mathrm{TeV}$ are presented.

Kruger 2010: Workshop on Discovery Physics at the LHC

December 5 - 10, 2010

Kruger National Park, Mpumalanga, South Africa 


\section{Introduction}

Although the standard model (SM) of fundamental particles and their interactions is in excellent agreement with most collider data, there are compelling reasons to believe new physics should appear at high energy scales. Some well-motivated theories of physics beyond the SM, including grand unified theories, composite models, technicolor, and superstring-inspired $E_{6}$ models, postulate the existence of a symmetry, beyond that of the SM, relating quarks and leptons and implying the existence of new bosons, called leptoquarks (LQ) [1]. An LQ carries color, has fractional electric charge, can have spin 0 (scalar) or spin 1 (vector), and couples to a lepton and a quark with coupling strength $\lambda$. An LQ would decay to a charged lepton and a quark, with an unknown branching fraction $\beta$, or a neutrino and a quark, with branching fraction $1-\beta$. Constraints from experiments sensitive to flavor-changing neutral currents, lepton-family-number violation, and other rare processes [2] favor LQs that couple to quarks and leptons within the same SM generation, for LQ masses accessible at current colliders. The first- (second-) generation scalar LQs studied here couple only to an electron (muon) or an electron-type (muon-type) neutrino and a light quark. Measurements at electron-proton colliders constrain the coupling $\lambda$ to be comparable to or less than the electromagnetic coupling $\lambda_{\mathrm{EM}} \equiv \sqrt{4 \pi \alpha_{\mathrm{EM}}} \approx 0.3$, for a first generation LQ mass, $M_{\mathrm{LQ}}$, less than $300 \mathrm{GeV}[3,4]$. Prior to this work, the DØ Collaboration set the most stringent limit for a broad range of the coupling $\lambda$ on the mass of the first- and second-generation scalar LQs, namely, $M_{\mathrm{LQ}}>299 \mathrm{GeV}[5]$ and $M_{\mathrm{LQ}}>316 \mathrm{GeV}$ [6] for $\beta=1$.

This communication presents the results of a search for pair production of first- and secondgeneration scalar LQs using events containing two charged leptons (electrons or muons, respectively) and two jets (the experimental signature of the hadronization of partons) from a data sample of $p p$ collisions at $\sqrt{s}=7 \mathrm{TeV}$ collected in 2010 with the Compact Muon Solenoid (CMS) detector at the LHC. The data samples correspond to an integrated luminosity of $33.2 \pm 3.7 \mathrm{pb}^{-1}$ and $34.0 \pm 3.7 \mathrm{pb}^{-1}$ for the first- and second-generation search, respectively. In $p p$ collisions at this energy, LQs are predominantly produced in pairs via gluon-gluon fusion and quark-antiquark annihilation with a cross section that depends on the strong coupling constant $\alpha_{\mathrm{s}}$ but is nearly independent of $\lambda$. This cross section depends on the spin and the mass of the LQ and, for scalar LQs, has been calculated including Next-to-Leading-Order (NLO) Quantum Chromodynamics (QCD) corrections [7]. In this study we did not consider possible contributions from single LQ production, which has a cross section that is dependent on $\lambda$.

The CMS experiment is described elsewhere [8]. Online, events to be used in the first- and second-generation LQ analysis are collected, respectively, with an efficiency greater than $99.9 \%$ by single and double electron triggers, and with an efficiency of approximately $92 \%$ by a single muon trigger. Offline, an initial sample containing at least two electrons (muons) and at least two jets is selected for the first- (second-) generation LQ analysis. The two leading (in $p_{\mathrm{T}}$ ) electrons or muons, and two leading jets are used in the analyses and are all required to have $p_{\mathrm{T}}>30 \mathrm{GeV}$. Selected electrons, muons and jets have pseudorapidities $|\eta|<2.5,|\eta|<2.4$ and $|\eta|<3.0$, respectively. Details on the reconstruction of electrons, muons and jets, and additional selection requirements for the first- and second-generation LQ analyses can be found in [9] and [10].

Preliminary requirements $M_{11}>50 \mathrm{GeV}$ and $S_{\mathrm{T}}>250 \mathrm{GeV}$ are applied, where $M_{11}$ is the dilepton invariant mass and $S_{\mathrm{T}}$ is defined as the sum of the magnitudes of the $p_{\mathrm{T}}$ of the two leading 
leptons and two leading jets. At this stage of the selection, referred to as pre-selection, there are sufficient data to compare with Monte Carlo (MC) generated events, where the response of the detector was simulated using GEANT4 [11]. Good data-MC agreement is observed in the shape of all kinematic distributions of the selected leptons and jets. The dominant SM backgrounds are $\mathrm{Z} / \gamma^{*}+\mathrm{jets}$ and $t \bar{t}$, which are simulated and estimated using, respectively, ALPGEN [12] and MAdGRAPH [13, 14] interfaced with PYTHIA [15] for parton showering and hadronization. The $\mathrm{Z} / \gamma^{*}+$ jets MC has been normalized to the data at the Z-boson peak at pre-selection level, where the $\mathrm{Z} / \gamma^{*}+$ jets background dominates the sample. The $t \bar{t} \mathrm{MC}$ has been normalized to the CMS measurement of the $t \bar{t}$ cross section [16]. Other backgrounds include multijet production, which is estimated from data and found negligible for both analyses. There is also a small contribution from di-boson and single top production, determined from MC. Signal samples for LQ masses 200-500 $\mathrm{GeV}$ were generated with PYTHIA [15], version 6.422, tune D6T [17, 18].

To reduce the background from $\mathrm{Z} / \gamma^{*}+$ jets production, a minimal value of $M_{11}$ well above the mass of the $\mathrm{Z}$ boson is required, and, to reduce all SM backgrounds, $S_{\mathrm{T}}$ is required to be large. While the LQ signal is expected to appear as a peak in the mass distribution of the lepton-jet pairs, we find that the $S_{T}$ variable is more powerful with the present statistics as it is not affected by combinatorics. The minimal values required for $M_{11}$ and $S_{\mathrm{T}}$ were optimized by minimizing the expected upper limit on the leptoquark cross section in the absence of an observed signal using a Bayesian approach $[19,20]$ that is well suited for counting experiments in the Poisson regime. The optimized lower value of $M_{\mathrm{ee}}\left(M_{\mu \mu}\right)$ is found to be $125 \mathrm{GeV}(115 \mathrm{GeV})$ for all the LQ hypotheses under test, while the lower value of $S_{\mathrm{T}}$ varies as indicated in Table 1. Fig. 1 shows the $S_{\mathrm{T}}$ distributions. Table 1 shows the number of surviving events for MC signal, MC background and data samples after applying the full, optimized selection. The reported product of signal selection efficiency and acceptance is estimated from MC simulated events. The product of the di-lepton efficiency and acceptance, prior to any $M_{11}$ and jet requirements, is $59-68 \%$ and $61-70 \%$ for the first- and second-generation LQ analysis for LQ masses of 200-500 GeV.
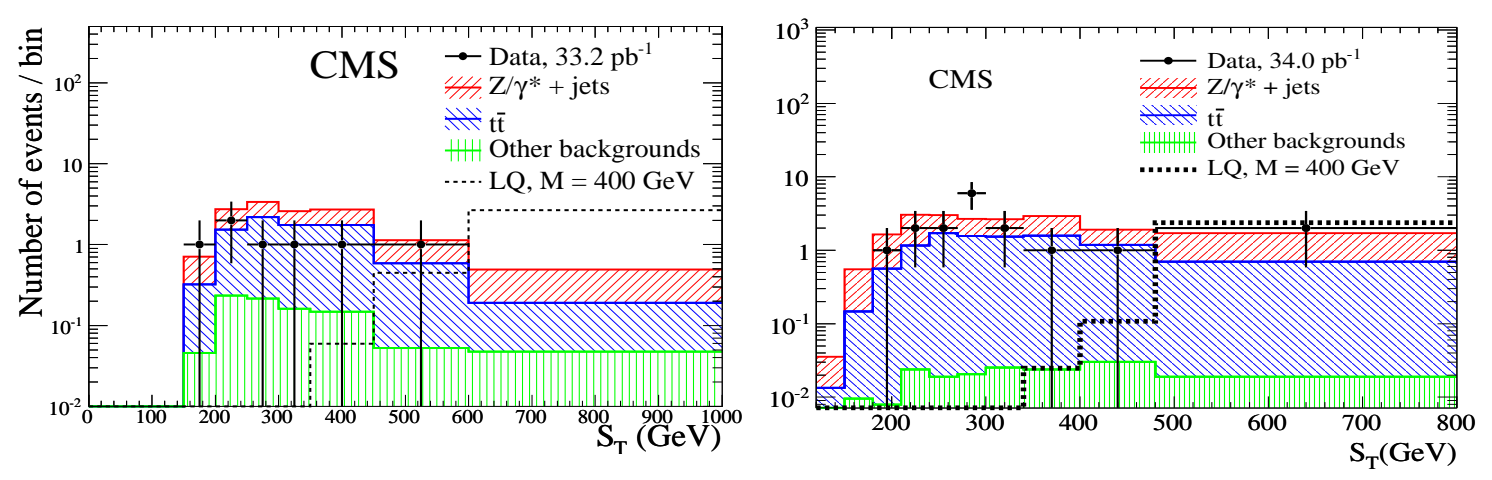

Figure 1: The $S_{\mathrm{T}}$ distribution for events that have passed the final selection requirement, except the requirement on $S_{\mathrm{T}}$ itself, for the first- (left) and second-generation (right) LQ analyses. The $\mathrm{Z} / \gamma^{*}+$ jets and $t \bar{t}$ contributions are rescaled by normalization factors determined from data. Other backgrounds include $\mathrm{W}+\mathrm{jets}$, di-boson, and single top. All background histograms are cumulative.

The systematic uncertainties affecting the estimated number of signal events are $15 \%$ for both 
Table 1: Number of events for MC LQ signal (for $\beta=1$ ), MC background, and data samples after the final analysis selections for the first- (top section) and second-generation (bottom section) LQ searches. The product of signal acceptance and efficiency is also reported for different LQ masses. The $\mathrm{Z} / \gamma^{*}+\mathrm{jets}$ and $t \bar{t}$ contributions are rescaled by normalization factors determined from data. Other backgrounds include $W+$ jets, di-boson, and single top. The uncertainties reported here are from MC statistics. Systematic uncertainties are discussed in the text. The observed and expected 95\% C.L. upper limit (u.l.) on the leptoquark pair production cross section $\sigma$ are shown in the last column.

\begin{tabular}{|c|c|c|c|c|c|c|c|c|}
\hline \multirow{3}{*}{$\begin{array}{c}M_{\mathrm{LQ}} \\
\left(S_{\mathrm{T}} \mathrm{Cut}\right) \\
{[\mathrm{GeV}]}\end{array}$} & \multicolumn{2}{|c|}{ Signal Samples (MC) } & \multicolumn{4}{|c|}{ Standard Model Background Samples (MC) } & \multirow{3}{*}{$\begin{array}{l}\text { Evts } \\
\text { in } \\
\text { Data }\end{array}$} & \multirow{3}{*}{$\begin{array}{c}\text { Obs./Exp. } \\
\text { 95\% C.L. } \\
\text { u.l. on } \sigma[\mathrm{pb}]\end{array}$} \\
\hline & Selected & Acceptance & & Selecte & Events in & & & \\
\hline & Events & $\times$ Efficiency & $t \bar{t}+$ jets & $\mathrm{Z} / \gamma^{*}+$ jets & Others & All & & \\
\hline $200\left(S_{T}>340\right)$ & $117.5 \pm 0.8$ & $0.297 \pm 0.002$ & $2.6 \pm 0.1$ & $2.0 \pm 0.2$ & $0.27 \pm 0.05$ & $4.9 \pm 0.2$ & 2 & $0.441 / 0.720$ \\
\hline $300\left(S_{T}>470\right)$ & $17.3 \pm 0.09$ & $0.430 \pm 0.002$ & $0.52 \pm 0.05$ & $0.75 \pm 0.07$ & $0.10 \pm 0.02$ & $1.4 \pm 0.1$ & 1 & $0.292 / 0.332$ \\
\hline $400\left(S_{T}>560\right)$ & $3.55 \pm 0.02$ & $0.522 \pm 0.002$ & $0.20 \pm 0.03$ & $0.41 \pm 0.05$ & $0.06 \pm 0.02$ & $0.67 \pm 0.07$ & 1 & $0.257 / 0.234$ \\
\hline $500\left(S_{T}>660\right)$ & $0.87 \pm 0.00$ & $0.565 \pm 0.002$ & $0.08 \pm 0.02$ & $0.23 \pm 0.05$ & $0.02 \pm 0.01$ & $0.33 \pm 0.05$ & 0 & $0.166 / 0.194$ \\
\hline $200\left(S_{T}>310\right)$ & $160 \pm 20$ & $0.388 \pm 0.003$ & $4.6 \pm 0.1$ & $4.08 \pm 0.07$ & $0.10 \pm 0.01$ & $8.8 \pm 0.2$ & 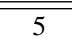 & $0.438 / 0.695$ \\
\hline $300\left(S_{T}>440\right)$ & $21 \pm 2$ & $0.518 \pm 0.004$ & $1.15 \pm 0.07$ & $1.53 \pm 0.03$ & $0.04 \pm 0.01$ & $2.7 \pm 0.1$ & 3 & $0.335 / 0.326$ \\
\hline $400\left(S_{T}>560\right)$ & $4.0 \pm 0.4$ & $0.578 \pm 0.004$ & $0.31 \pm 0.04$ & $0.67 \pm 0.01$ & $0.01 \pm 0.004$ & $0.99 \pm 0.04$ & 1 & $0.219 / 0.222$ \\
\hline $500\left(S_{T}>700\right)$ & $0.9 \pm 0.1$ & $0.602 \pm 0.004$ & $0.09 \pm 0.02$ & $0.28 \pm 0.01$ & $0.003 \pm 0.002$ & $0.37 \pm 0.02$ & 0 & $0.152 / 0.180$ \\
\hline
\end{tabular}

analyses, and are dominated by the uncertainty on the integrated luminosity, and lepton selection and reconstruction efficiencies. The systematic uncertainties for the background are $28 \%$ and $26 \%$ for first- and second-generation LQ analysis, and are dominated by the statistical uncertainty on the data for the normalization of the $\mathrm{Z} / \gamma^{*}+\mathrm{jets}$ and $t \bar{t} \mathrm{MC}$, the uncertainty on the jet energy scale, and the uncertainty on the MC modeling of the shape of the $\mathrm{Z} / \gamma^{*}+$ jets background.

The numbers of observed events in the collision data samples that pass the selection criteria optimized for each LQ mass considered are consistent with the predictions from SM processes, as reported in Table 1. An upper limit on the LQ cross section in the absence of signal is therefore set using a Bayesian approach [20] that uses a Poisson likelihood, a flat prior for the signal cross section, and log-normal priors for the parameters used to model the systematic uncertainties. Fig. 2 shows the 95\% Confidence Level (C.L.) upper limit on the LQ pair production cross section times $\beta^{2}$ as a function of the leptoquark mass. The systematic uncertainties are included in the calculation. The upper limits are compared to an NLO prediction of the LQ pair production cross section [7] to set an observed 95\% C.L. exclusion on LQ masses smaller than $384 \mathrm{GeV}$ (expected $391 \mathrm{GeV}$ ) and $394 \mathrm{GeV}$ (expected $394 \mathrm{GeV}$ ), assuming $\beta=1$, for first- and second-generation LQs. Fig. 3 shows the minimum $\beta$ for a $95 \%$ C.L. exclusion of the LQ hypothesis as a function of LQ mass.

In conclusion, searches for pair production of first- and second-generation scalar leptoquarks have been performed using $7 \mathrm{TeV} p p$ collision data corresponding to an integrated luminosity of 33.2 and $34.0 \mathrm{pb}^{-1}$, respectively. The numbers of observed candidate events agree well with the numbers of expected standard model background events. A Bayesian approach that includes the treatment of systematic uncertainties as nuisance parameters is used to set limits on the LQ cross section times $\beta^{2}$ as a function of LQ mass, where $\beta$ is the leptoquark branching fraction into a charged lepton and a quark. By comparing these upper limits to a theoretical calculation of the LQ 

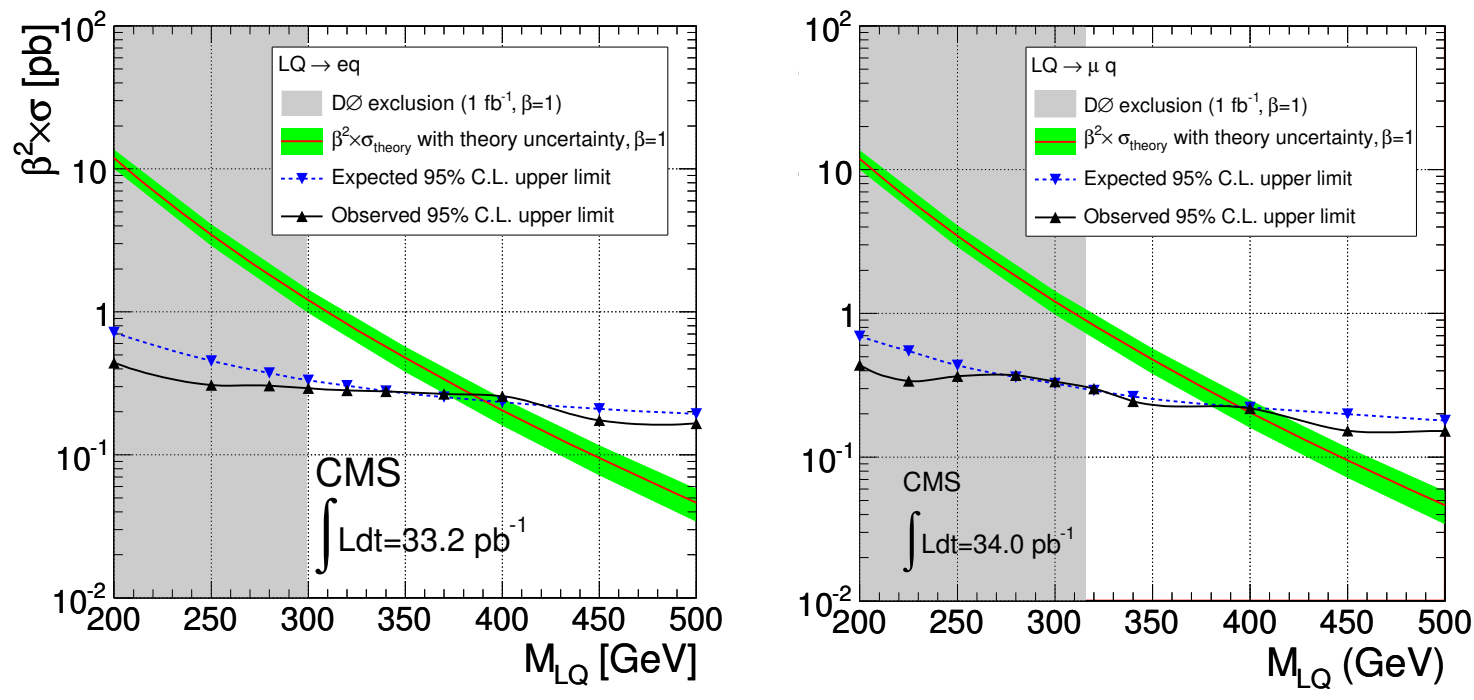

Figure 2: The expected and observed upper limit at 95\% C.L. on the LQ pair production cross section times $\beta^{2}$ as a function of the LQ mass for the first- (left) and second-generation (right) LQ searches. The systematic uncertainties are included in the calculation. The shaded region is excluded by the current $\mathrm{D} \varnothing$ limit for $\beta=1$. The $\sigma_{\text {theory }}$ curve and its band represent, respectively, the theoretical LQ pair production cross section and the uncertainties due to the choice of PDF and renormalization/factorization scales [7].
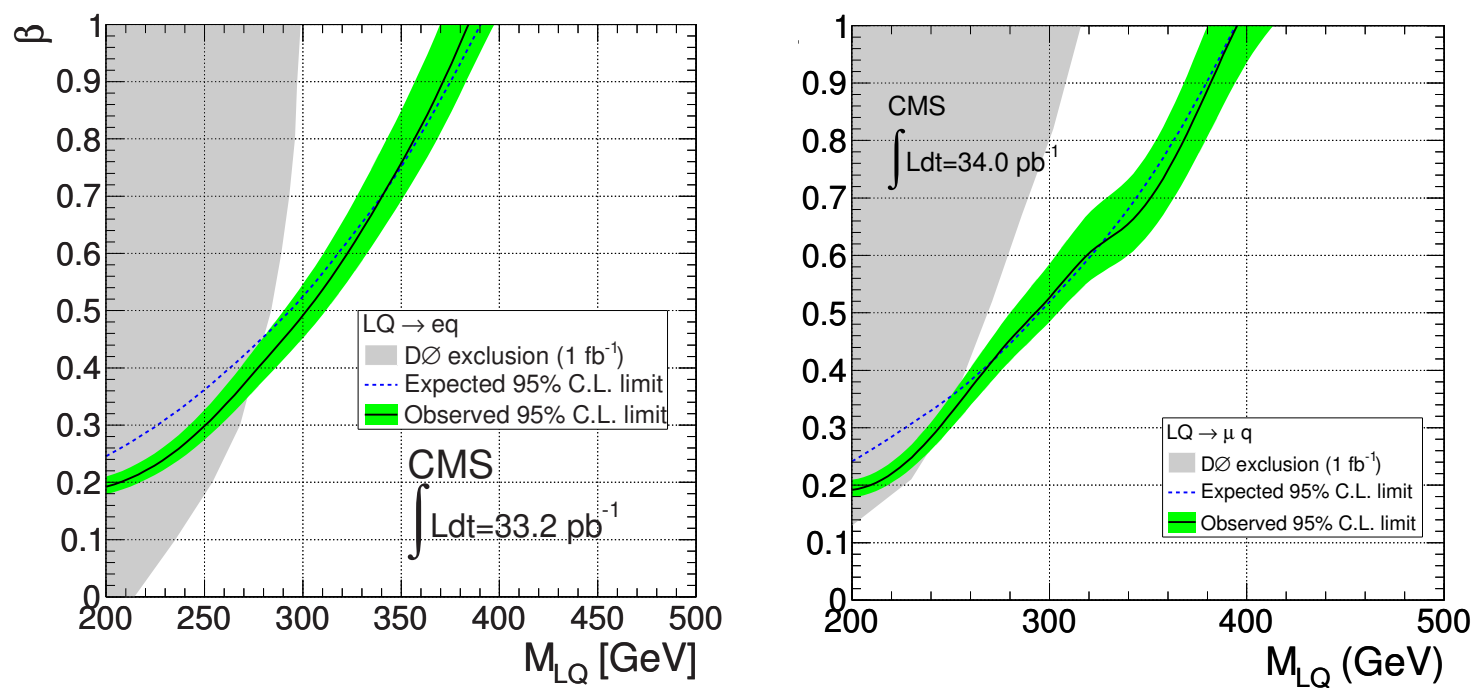

Figure 3: Minimum $\beta$ for a 95\% C.L. exclusion of the LQ hypothesis as a function of LQ mass for the first- (left) and second-generation (right) LQ searches. The observed (expected) exclusion curve is obtained using the observed (expected) upper limit and the central value of the theoretical LQ pair production cross section. The band around the observed exclusion curve is obtained by considering the observed upper limit while taking into account the uncertainties on the theoretical cross section. The shaded region is excluded by the current D $\varnothing$ limits, which combines results from searches in the two lepton and lepton-neutrino channels (and, for the first-generation case, also the two neutrino channel). 
pair production cross section, the existence of first- and second-generation scalar LQ with masses below $384 \mathrm{GeV}$ and $394 \mathrm{GeV}$ for $\beta=1$ has been excluded at $95 \%$ C.L., with corresponding cross section limits of $0.265 \mathrm{pb}$ and $0.223 \mathrm{pb}$. The lower limits on the LQ masses set for values of $\beta$ larger than about 0.4 are the most restrictive direct limits to date.

\section{References}

[1] Masahiro Kuze and Yves Sirois. Search for particles and forces beyond the standard model at HERA ep and Tevatron p anti-p colliders. Prog.Part.Nucl.Phys., 50:1, 2003.

[2] W. Buchmuller and D. Wyler. Constraints on SU(5) Type Leptoquarks. Phys. Lett., B177:377, 1986.

[3] H1 Collaboration (A. Aktas et al.). Search for leptoquark bosons in e p collisions at HERA. Phys. Lett., B629:9, 2005.

[4] ZEUS Collaboration (S. Chekanov et al.). A search for resonance decays to lepton + jet at HERA and limits on leptoquarks. Phys. Rev., D68:052004, 2003.

[5] DØ Collaboration (V. M. Abazov et al.). Search for pair production of first-generation leptoquarks in $p \bar{p}$ collisions at sqrt(s)=1.96 TeV. Phys. Lett., B681:224, 2009.

[6] DØ Collaboration (V. M. Abazov et al.). Search for pair production of second generation scalar leptoquarks. Phys.Lett., B671:224, 2009.

[7] M. Krämer et al. Pair production of scalar leptoquarks at the CERN LHC. Phys. Rev., D71:057503, 2005; and private communications.

[8] CMS Collaboration (S. Chatrchyan et al.). The CMS experiment at the CERN LHC. JINST, 0803:S08004, 2008.

[9] CMS Collaboration (V. Khachatryan et al.). Search for Pair Production of First-Generation Scalar Leptoquarks in pp Collisions at sqrt(s) $=7 \mathrm{TeV}$. 2010. [arXiv:1012.4031 [hep-ex]], accepted by PRL.

[10] CMS Collaboration (V. Khachatryan et al.). Search for Pair Production of Second-Generation Scalar Leptoquarks in pp Collisions at sqrt(s) $=7 \mathrm{TeV}$. 2010. [arXiv:1012.4033 [hep-ex]], accepted by PRL.

[11] S. Agostinelli et al. Geant4: A simulation toolkit. Nucl. Instrum. and Methods, A506:250, 2003.

[12] M.L. Mangano et al. ALPGEN, a generator for hard multiparton processes in hadronic collisions. JHEP, 07:001, 2003.

[13] F. Maltoni and T. Stelzer. Madevent: Automatic event generation with madgraph. JHEP, 02:027, 2003.

[14] J. Alwall et al. MadGraph/MadEvent v4: The new web generation. JHEP, 09:028, 2007.

[15] S. Mrenna T. Sjöstrand and P. Skands. PYTHIA 6.4 physics and manual. JHEP, 05:026, 2006.

[16] CMS Collaboration (V. Khachatryan et al.). First Measurement of the Cross Section for Top-Quark Pair Production in Proton-Proton Collisions at $\sqrt{s}=7 \mathrm{TeV}$. Submitted to Physics Letters B, 2010.

[17] Rick Field. Physics at the Tevatron. Acta Phys. Polon., B39:2611, 2008.

[18] R. Field. Studying the underlying event at CDF and the LHC. In P. Bartalini and L. Fanó, editors, Proceedings of the First International Workshop on Multiple Partonic Interactions at the LHC MPI'08, October 27-31, 2008, Perugia, Italy, October 2009.

[19] K. Nakamura et al. The Review of Particle Physics - Section 33.3.1. J. Phys., G37:075021, 2010.

[20] I. Bertram, G. Landsberg, J. Linnemann, R. Partridge, M. Paterno, and H.B. Prosper. A Recipe for the construction of confidence limits. Technical report, 2000. FERMILAB-TM-2104. 